\title{
Peripheral nervous system insulin resistance in ob/ob mice
}

\author{
Caleb W Grote ${ }^{1}$, Anna L Groover ${ }^{1}$, Janelle M Ryals ${ }^{1}$, Paige C Geiger ${ }^{2}$, Eva L Feldman ${ }^{3}$ and Douglas E Wright ${ }^{*}$
}

\begin{abstract}
Background: A reduction in peripheral nervous system (PNS) insulin signaling is a proposed mechanism that may contribute to sensory neuron dysfunction and diabetic neuropathy. Neuronal insulin resistance is associated with several neurological disorders and recent evidence has indicated that dorsal root ganglion (DRG) neurons in primary culture display altered insulin signaling, yet in vivo results are lacking. Here, experiments were performed to test the hypothesis that the PNS of insulin-resistant mice displays altered insulin signal transduction in vivo. For these studies, nondiabetic control and type 2 diabetic ob/ob mice were challenged with an intrathecal injection of insulin or insulin-like growth factor 1 (IGF-1) and downstream signaling was evaluated in the DRG and sciatic nerve using Western blot analysis.

Results: The results indicate that insulin signaling abnormalities documented in other "insulin sensitive" tissues (i.e. muscle, fat, liver) of ob/ob mice are also present in the PNS. A robust increase in Akt activation was observed with insulin and IGF-1 stimulation in nondiabetic mice in both the sciatic nerve and DRG; however this response was blunted in both tissues from ob/ob mice. The results also suggest that upregulated JNK activation and reduced insulin receptor expression could be contributory mechanisms of PNS insulin resistance within sensory neurons.
\end{abstract}

Conclusions: These findings contribute to the growing body of evidence that alterations in insulin signaling occur in the PNS and may be a key factor in the pathogenesis of diabetic neuropathy.

Keywords: Diabetic neuropathy, Neuronal insulin resistance, Neurotrophic support

\section{Background}

Diabetes and metabolic syndrome are risk factors for several neurological diseases, and emerging evidence has indicated that neuronal insulin resistance may be involved in disease pathogenesis [1]. While altered insulin signaling is known to be the key factor in the development of diabetes, the role that it plays in diabetic neuropathy (DN) is not well understood. However, it has been demonstrated that neuronally-targeted insulin treatment can improve signs of neuropathy without altering blood glucose levels [2-4]. Recent evidence suggests that cultured sensory neurons from insulinresistant mice display classic signs of insulin resistance and that insulin resistance may be contributing to mitochondrial dysfunction and increased ROS in DN [5-7].

\footnotetext{
* Correspondence: dwright@kumc.edu

'Department of Anatomy and Cell Biology, the University of Kansas Medical Center, Kansas City, KS 66160, USA

Full list of author information is available at the end of the article
}

Furthermore, clinical evidence has reported that insulin resistance appears to be an independent risk factor for both autonomic and peripheral neuropathy [8].

Although neurons do not appear to rely on insulin for glucose uptake [9], insulin does have an important role in both the CNS and PNS. Insulin promotes in vivo nerve regeneration $[4,10,11]$, induces neurite outgrowth [12,13], maintains neuronal mitochondrial function [14,15], supports memory formation $[16,17]$, and regulates hypothalamic metabolic control $[18,19]$. While the exact mechanisms through which insulin promotes these functions remain unclear, insulin is considered a potent neurotrophic factor key to maintaining proper neuronal function.

Insulin and insulin-like growth factor 1 (IGF-1) signaling is propagated by phosphorylation events that begin with the intrinsic tyrosine kinase activity of the insulin or IGF receptor (reviewed in $[20,21]$ ) and continue with subsequent activation of both the PI3K-Akt and MAPK cascades. While these pathways are well defined in 
muscle, adipose, and liver, insulin signaling and its actions in the PNS are poorly understood.

In an insulin-resistant state, the cellular effects of insulin are blunted due to improper signal propagation resulting from several different mechanisms, including 1) degradation of the insulin receptor [22-25], 2) removal of key tyrosine phosphorylation sites by overactivation of protein tyrosine phosphatases [26-29], and 3) increased phosphorylation at inhibitory IRS serine residues due to elevated stress kinases, such as JNK [30-35]. However, the extent to which these mechanisms affect insulin signal transduction in the PNS is not clear.

Growing evidence suggests that neurons may become insulin resistant similar to other tissues. However, no in vivo evidence of PNS insulin resistance has been presented, and the cellular mechanisms associated with PNS insulin resistance have not been thoroughly investigated. Here, we demonstrate that the DRG and sciatic nerve of $o b / o b$ mice display reduced insulin signaling in response to an intrathecal injection of insulin.
Furthermore, the PNS of $o b / o b$ mice has alterations in cellular mechanisms of insulin resistance, including decreased DRG insulin receptor expression and upregulation of JNK activity in the sciatic nerve.

\section{Results}

Insulin resistance in $o b / o b$ mice

To quantify the extent of systemic insulin resistance in $o b / o b$ mice, nondiabetic and diabetic $o b / o b$ mice underwent an IPGTT at 9 weeks of age (Figure 1A). Blood glucose levels of the $o b / o b$ mice were significantly higher than nondiabetic mice throughout the course of the experiment and the area under the curve (AUC) was also significantly elevated for $o b / o b$ mice (Figure 1B). Results from the ITT indicated that nondiabetic, insulin-injected mice exhibited an expected physiological decrease in blood glucose in response to insulin; however, $o b / o b$ mice displayed a transient elevation of glucose levels (Figure 1C). Statistical analysis of the data revealed that $o b / o b$ mice maintained elevated glucose levels
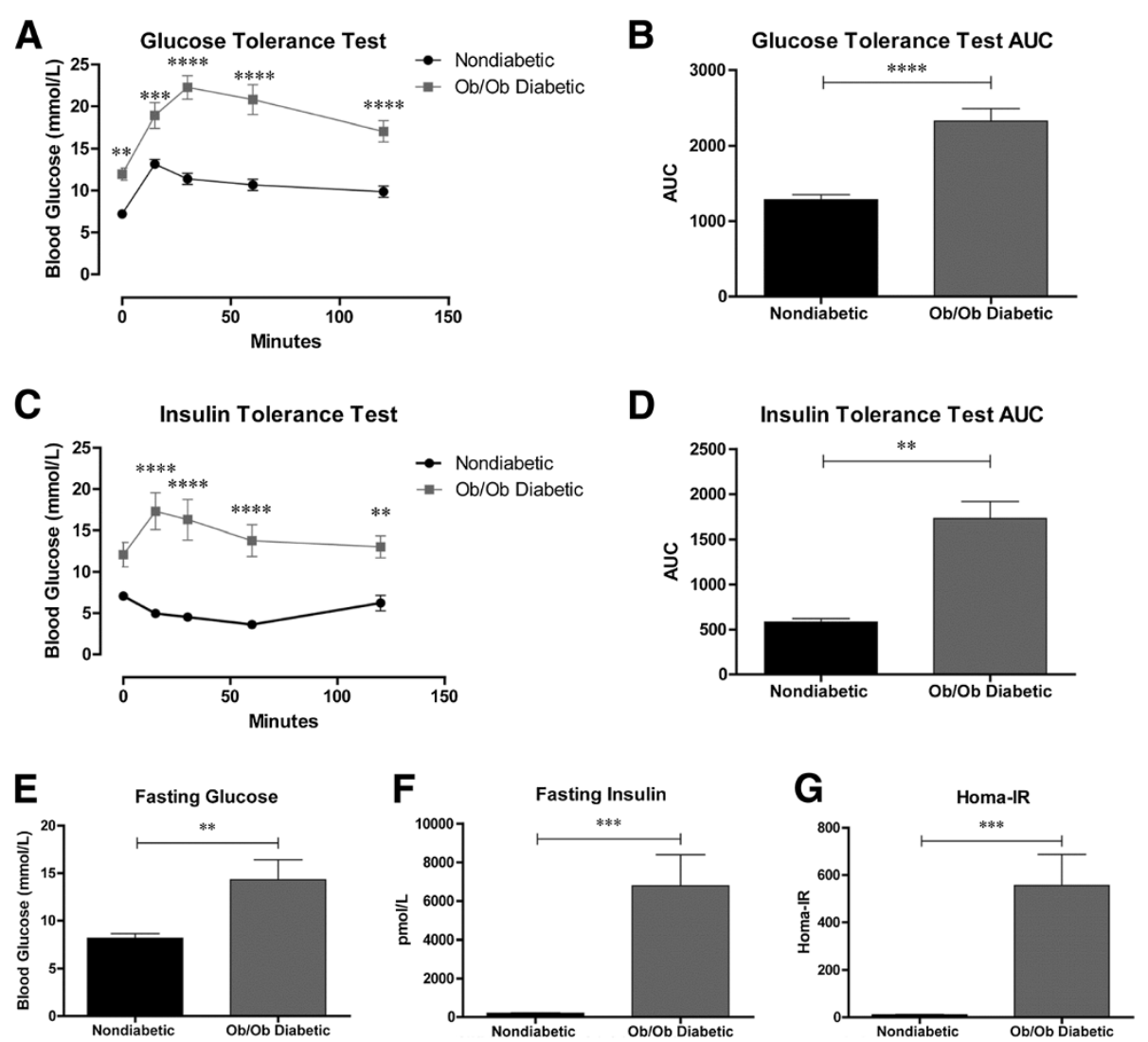

Figure $1 \mathrm{Ob} / \mathrm{ob}$ mice display classic signs of insulin resistance. A, B) An IPGTT showed significantly elevated blood glucose levels in ob/ob mice throughout the test. The blood glucose of ob/ob mice increased more than $10 \mathrm{mmol} / \mathrm{L}$ at its maximal level as opposed to nondiabetic mice that elevated less than $6 \mathrm{mmol} / \mathrm{L}$ after glucose injection, indicating severe glucose intolerance in ob/ob mice. C, D) Similar to the IPGTT, data from the ITT showed reduced insulin sensitivity in ob/ob mice. In fact, an insulin dose of $1.5 \mathrm{U} / \mathrm{Kg}$ did not decrease the blood glucose level of ob/ob mice, whereas this dose lowered the blood glucose of nondiabetic controls by approximately $3.6 \mathrm{mmol} / \mathrm{L}$. E-G) At 10 weeks of age, ob/ob mice had significantly elevated blood glucose and serum insulin levels. Accordingly, the HOMA-IR measure of insulin resistance was significantly higher in ob/ob mice. ${ }^{* *}=p<0.01,{ }^{* * *}=p<0.001,{ }^{* * *}=p<0.0001$. IPGTT $n=7$ nondiabetic mice, $n=6$ ob/ob. ITT $n=4$ nondiabetic mice, $n=4$ ob/ob. 
compared to nondiabetic controls throughout most of the study, and that the AUC was significantly higher for diabetic $o b / o b$ mice (Figure 1D). The HOMA-IR, a measure of insulin resistance, was calculated using fasting blood glucose and serum insulin levels from 10 week old mice. $\mathrm{Ob} / \mathrm{ob}$ mice had significantly higher blood glucose levels $(14.3 \pm 2.1 \mathrm{mmol} / \mathrm{L})$ compared to nondiabetic mice $(8.2 \pm 0.5 \mathrm{mmol} / \mathrm{L})$ (Figure 1E). Fasting insulin levels were also significantly higher in diabetic $o b / o b$ mice $(6780 \pm$ $1610 \mathrm{pmol} / \mathrm{L})$ compared to nondiabetic mice (198 \pm 25 $\mathrm{pmol} / \mathrm{L}$, Figure 1F). As such, $o b / o b$ mice had a significantly elevated HOMA-IR as compared to nondiabetic mice $(557 \pm 130$ compared to $10.1 \pm 1.4$, respectively, Figure 1G). These results demonstrate significant glucose intolerance and insulin resistance in $o b / o b$ mice at this age.

\section{Mechanical allodynia in ob/ob mice}

To quantify a known behavioral abnormality associated with neuropathy in mice, mechanical sensitivity was assessed in nondiabetic and diabetic ob/ob mice at 8, 9, 10 , and 11 weeks of age. There were no differences in mechanical thresholds between nondiabetic and $o b / o b$ diabetic mice at 8,9 , or 10 weeks of age. However, at 11 weeks, there was a significant decrease in the mechanical thresholds of diabetic $o b / o b$ mice compared to nondiabetic mice (Figure 2), consistent with sensory aberrations associated with peripheral neuropathy as previously reported in this genetic mouse strain [36].

\section{Blunted insulin and IGF-1 Akt activation in ob/ob DRG and sciatic nerve}

Insulin stimulation causes a robust activation of Akt in insulin-sensitive tissues like muscle and adipose, as well as in neurons of both the peripheral and central nervous systems. Moreover, reduced insulin-induced activation of

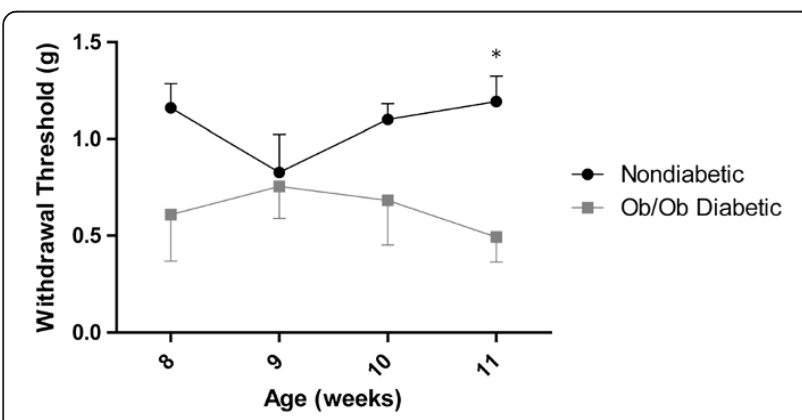

Figure $2 \mathrm{Ob} / \mathrm{ob}$ mice develop mechanical allodynia. Mechanical thresholds were tested using von Frey monofilaments at 8, 9, 10, and 11 weeks of age. Ob/Ob mice did not display significant differences from nondiabetic controls at 8, 9, or 10 weeks. However, at week 11, ob/ob mice had a significant decrease in their mechanical withdrawal thresholds. ${ }^{*}=p<0.05 . n=6$ nondiabetic mice, $n=6$ ob/ob diabetic mice.
Akt is a hallmark of insulin resistance $[5,6,37,38]$. Here, nondiabetic and diabetic $o b / o b$ mice were administered either intrathecal PBS or insulin and the DRG and sciatic nerve were harvested 30 minutes later for Western blot analysis to assess Akt activation. Both nondiabetic control and $o b / o b$ mice display significantly elevated blood glucose levels following intrathecal injection of PBS. Nondiabetic mice glucose levels increased from $6.6 \pm 0.4 \mathrm{mmol} / \mathrm{L}$ to $8.6 \pm 0.5 \mathrm{mmol} / \mathrm{L}$, whereas $o b / o b$ levels increased from $12.1 \pm 2.4 \mathrm{mmol} / \mathrm{L}$ to $22.3 \pm 2.4 \mathrm{mmol} / \mathrm{L}$. Glucose levels in nondiabetic mice significantly decreased from $7.0 \pm 0.3 \mathrm{mmol} / \mathrm{L}$ to $3.3 \pm 0.3 \mathrm{mmol} / \mathrm{L}$ after intrathecal insulin injection. $\mathrm{Ob} / \mathrm{ob}$ mice glucose levels $30 \mathrm{mi}-$ nutes after insulin injection were not significantly different from baseline, starting at $12.7 \pm 0.9 \mathrm{mmol} / \mathrm{L}$ and ending at $12.3 \pm 1.5 \mathrm{mmol} / \mathrm{L}$ after 30 minutes.

In nondiabetic mice, insulin produced a strong elevation in levels of activated Akt (p(ser473)Akt/total Akt) in both the DRG and sciatic nerve (Figure 3A,B). However in $o b / o b$ mice, Akt activation was significantly lower in the DRG and sciatic nerve. In fact, insulin failed to significantly increase Akt activation over baseline in the DRG of $o b / o b$ mice. For comparison, Akt activation in the DRG was increased 3.1 fold in nondiabetic mice and 1.6 fold in $o b / o b$ diabetic mice. In the sciatic nerve, insulin produced 9.7 and 6.1 fold increase in Akt activation in nondiabetic and $o b / o b$ mice, respectively.

To confirm that these results were not dependent on the intrathecal route of delivery, a small number of mice were administered intraperitoneal insulin at a dose of $3.33 \mathrm{U} / \mathrm{kg}$. PBS injections once again appeared to cause an increase in blood glucose levels from baseline, nondiabetic mice levels started at $7.0 \pm 1.4 \mathrm{mmol} / \mathrm{L}$ and ended at $8.6 \pm$ $1.4 \mathrm{mmol} / \mathrm{L}(\mathrm{p}>0.05)$, whereas $o b / o b$ mice showed a significant increase from $10.8 \pm 0.8 \mathrm{mmol} / \mathrm{L}$ to $15.4 \pm$ $1.0 \mathrm{mmol} / \mathrm{L}$. IP insulin resulted in significantly lower blood glucose levels in nondiabetic mice after 30 minutes, $7.8 \pm$ 0.6 versus $4.2 \pm 0.5 \mathrm{mmol} / \mathrm{L}$, respectively. $\mathrm{Ob} / \mathrm{ob}$ mice blood glucose levels were not significantly altered by IP insulin injection $15.3 \pm 3.0$ versus $13.6 \pm 3.9 \mathrm{mmol} / \mathrm{L}$. Similar to the intrathecal delivery route, a significant increase in Akt activation was observed in the DRG and sciatic nerve of nondiabetic mice stimulated with insulin; however, no significant change was observed in either tissue from $o b / o b$ mice. (Figure 4A,B). In the DRG, nondiabetic mice displayed a 2.4 fold change in Akt activation, compared to a 1.5 fold change in $o b / o b$ mice. IP insulin induced a 3.8 fold change in Akt in the sciatic nerve of nondiabetic mice, but only a 1.4 fold change in $o b / o b$ in the sciatic nerve from $o b / o b$ mice.

IGF-1 and insulin activate many of the same intracellular signaling pathways [21], and altered IGF-1 signaling has been demonstrated in states of insulin resistance [39]. Furthermore, IGF-1 resistance has recently been demonstrated 

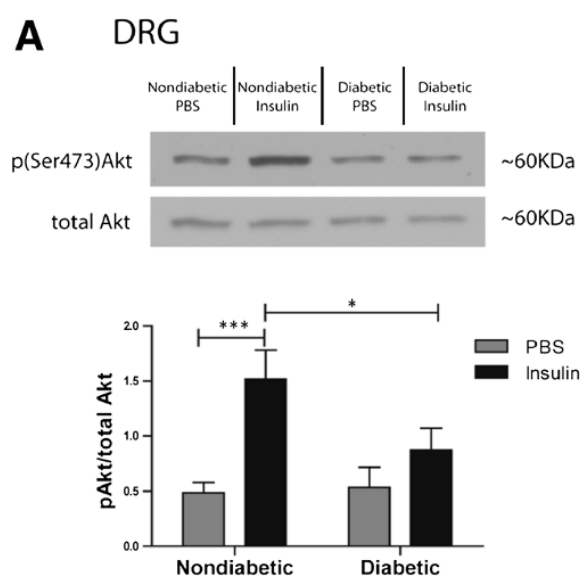

Figure 3 Intrathecal insulin-induced Akt activation is blunted in the PNS of $\boldsymbol{o b} / \mathbf{o b}$ mice. DRG (A) and sciatic nerve (B) were harvested after an intrathecal injection of PBS (nondiabetic $n=10, o b / o b n=7$ ) or insulin (nondiabetic $n=10, o b / o b n=9$ ) was administered to nondiabetic control and ob/ob mice. Nondiabetic mice displayed a robust and significant increase in Akt activation with insulin stimulation; however insulin failed to significantly activate Akt in the DRG of ob/ob mice. Furthermore, the maximal increase in Akt activation with insulin stimulation was significantly lower in both the DRG and sciatic nerve of ob/ob mice. There were no differences in mice that received PBS in either the DRG or sciatic nerve. ${ }^{*}=p<0.05,{ }^{* * *}=p<0.001$.

to be associated with brain insulin resistance and cognitive decline in Alzheimer's patients [40]. To investigate IGF-1 signal transduction in the PNS of $o b / o b$ mice, a dose of IGF-1 equimolar to $0.1 \mathrm{U}$ insulin was administered via an intrathecal injection. Blood glucose levels in both nondiabetic control and $o b / o b$ mice once again appeared to increase with intrathecal PBS injection, $7.7 \pm 0.3 \mathrm{mmol} /$ $\mathrm{L}$ at baseline as compared to $9.7 \pm 0.8 \mathrm{mmol} / \mathrm{L}(\mathrm{p}=0.056)$ after 30 minutes for nondiabetic mice and $12.1 \pm$ $1.9 \mathrm{mmol} / \mathrm{L}$ at baseline to $23.7 \pm 3.1 \mathrm{mmol} / \mathrm{L}$ after $30 \mathrm{mi}-$ nutes for $o b / o b$ mice. IT IGF-1 did not significantly alter blood glucose levels in nondiabetic mice, $9.3 \pm 0.4 \mathrm{mmol} / \mathrm{L}$
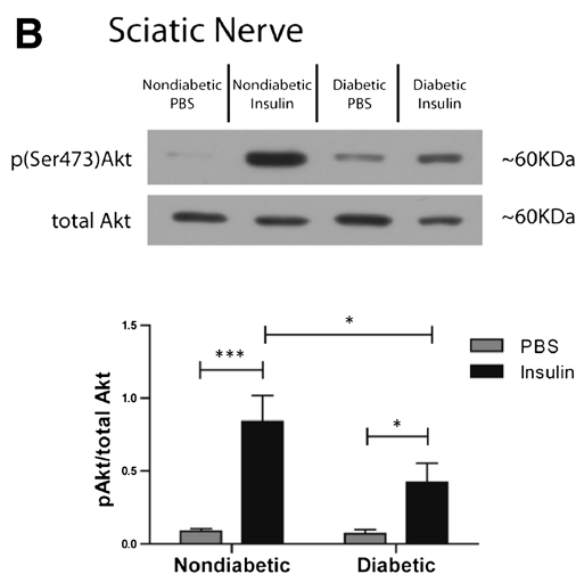

versus $8.1 \pm 0.3 \mathrm{mmol} / \mathrm{L}$. Ob/ob mice that received IT IGF1 had similar blood glucose profiles to $o b / o b$ mice that received IT PBS, with a significant increase in blood glucose after 30 minutes, $16.3 \pm 2.7 \mathrm{mmol} / \mathrm{L}$ as compared to $27.7 \pm$ $1.2 \mathrm{mmol} / \mathrm{L}$. Akt was significantly activated in the DRG from both nondiabetic (13.3 fold) and $o b / o b$ diabetic mice (6.0 fold). However, Akt activation was significantly lower in the DRG from $o b / o b$ mice compared to nondiabetic mice (Figure $5 \mathrm{~A}$ ). In the sciatic nerve of nondiabetic mice, IGF stimulation produced a significant 2.8 fold increase in Akt activation. In contrast, Akt was not significantly activated in the sciatic nerve of $o b / o b$ mice (Figure $5 \mathrm{~B}$ ).
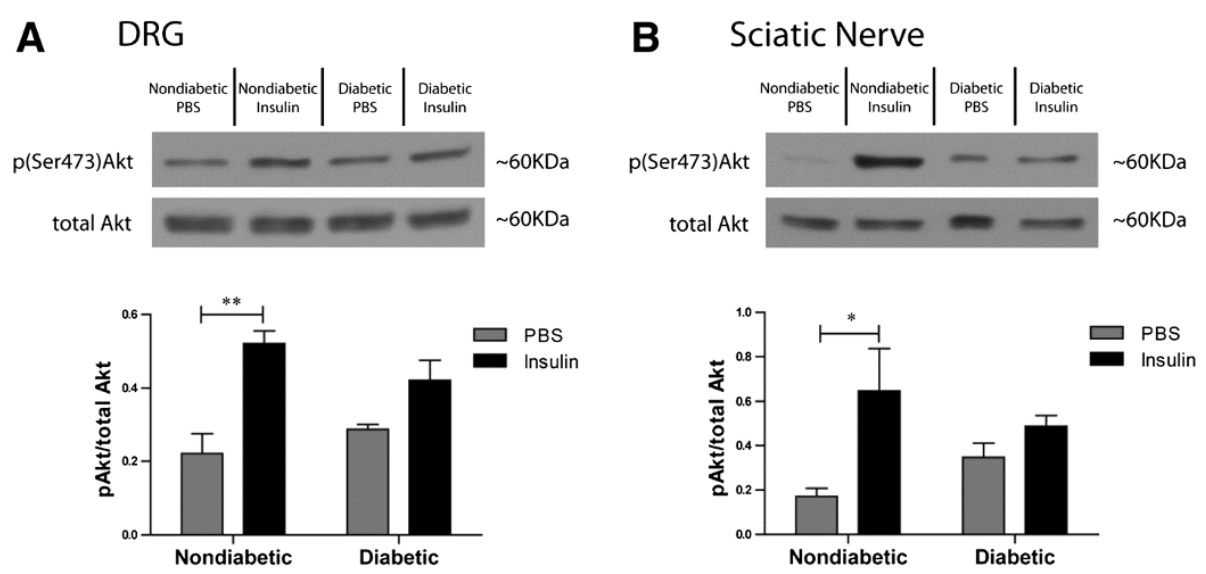

Figure 4 The PNS of $o b / o b$ mice showed reduced insulin-induced Akt activation in response to intraperitoneally-delivered insulin. Nondiabetic and ob/ob diabetic mice were given intraperitoneal injections of PBS (nondiabetic $n=3, o b / o b n=3$ ) or insulin at a dose of $3.33 \mathrm{U} / \mathrm{kg}$ (nondiabetic $\mathrm{n}=3$ and ob/ob $\mathrm{n}=3$ ). In both the DRG $(\mathbf{A})$ and sciatic nerve $(\mathbf{B})$ of nondiabetic mice, there was a significant increase in Akt activation in the insulin stimulated group as compared to mice that received PBS, yet no statistically significant changes were observed in the PNS from ob/ob mice. ${ }^{*}=p<0.05,{ }^{* *}=p<0.01$. 

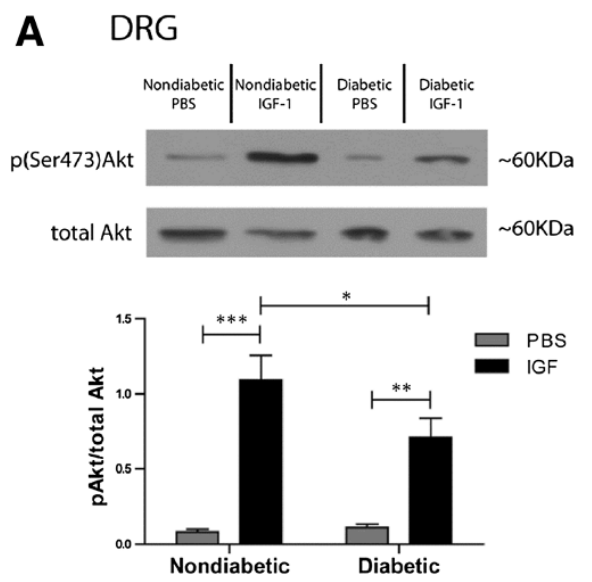
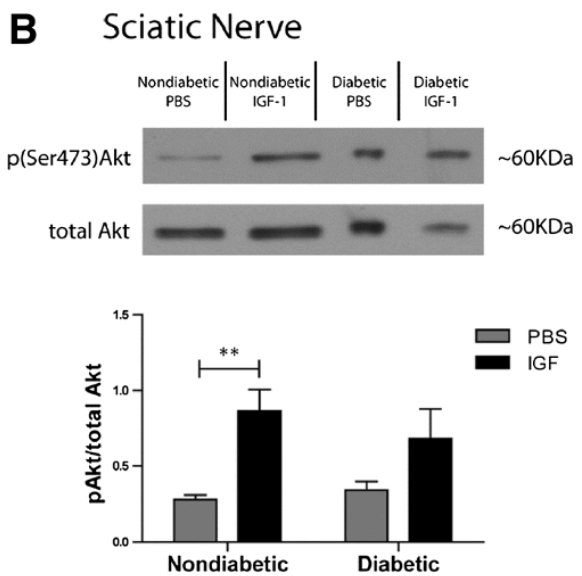

Figure 5 The PNS of $o b / o b$ mice displayed reduced Akt activation in response to intrathecal IGF-1. Similar to the results shown for intrathecal insulin, an intrathecal injection of IGF-1 produced a strong activation of Akt in both the DRG and sciatic nerve of nondiabetic mice, but the response was somewhat blunted in the PNS of ob/ob mice. In the DRG (A), there was a significant increase in Akt activation in both the nondiabetic and ob/ob mice; however, the activation level was significantly lower in the DRG from ob/ob mice. In the sciatic nerve (B), IGF-1 stimulation resulted in a significant Akt activation in nondiabetic mice, but not in the ob/ob mice. ${ }^{*}=p<0.05,{ }^{* *}=p<0.01,{ }^{* * *}=p<0.001 . n=8$ nondiabetic PBS, $\mathrm{n}=9$ nondiabetic IGF-1, $\mathrm{n}=7$ diabetic PBS, $\mathrm{n}=10$ diabetic IGF-1.

\section{Insulin signaling downstream of Akt in the DRG and} sciatic nerve

To assess whether diabetes-induced blunting of Akt activation was maintained downstream, several other insulin-responsive proteins were investigated via Western blot analysis, including mTor (protein synthesis), p70S6K (protein synthesis), AS160 (glucose uptake), and GSK3 $\beta$ (glycogen synthesis). At the insulin dose $(0.1 \mathrm{U})$ and time point (30 minute stimulation) that were investigated, no statistical differences $(p>0.05)$ were observed in the activation of these proteins even in control mice (Table 1). Additionally, similar to Akt results, no differences were observed in baseline levels between all proteins investigated. However, it is interesting to note that in both the DRG and sciatic nerve from $o b / o b$ mice, there is a consistent pattern of a reduced fold change in response to insulin as compared to responses in nondiabetic mice.
The PNS of $o b / o b$ mice display reduced insulin receptor expression and increased JNK activation

To explore possible mechanisms responsible for reduced PNS insulin sensitivity, we investigated several pathways known in other insulin-resistant tissues. One contributor to reduced insulin signaling is a downregulation of insulin receptor expression induced by hyperinsulinemia [23]. As shown in Figure 6A, protein levels of the insulin receptor subunit $\beta$ were significantly lower in the DRG of $o b / o b$ mice compared to nondiabetic mice. However, there was no statistical difference in the expression of insulin receptor between nondiabetic and $o b / o b$ mice in the sciatic nerve (Figure 6B). No significant differences between groups were observed in IGF-1 receptor expression in either the DRG or sciatic nerve (data not shown).

Our previous studies in primary DRG cultures reported an upregulation of IRS2 serine phosphorylation [6], a

Table 1 Downstream Akt pathway activation in the DRG and sciatic nerve after intrathecal insulin stimulation

\begin{tabular}{|c|c|c|c|c|}
\hline \multirow{3}{*}{$\begin{array}{l}\text { Protein of } \\
\text { interest }\end{array}$} & \multicolumn{2}{|c|}{ DRG } & \multicolumn{2}{|c|}{ Sciatic nerve } \\
\hline & Control nondiabetic & ob/ob diabetic & Control nondiabetic & ob/ob diabetic \\
\hline & Insulin-induced fold change & Insulin-induced fold change & Insulin-induced fold change & Insulin-induced fold change \\
\hline mTor & 1.52 & 1.00 & 1.13 & 0.98 \\
\hline AS160 & 1.47 & 1.28 & 2.13 & 1.22 \\
\hline p70s6K & 1.00 & 1.04 & 1.09 & 0.93 \\
\hline GSK3 $\beta$ & 1.26 & 1.17 & 1.56 & 0.88 \\
\hline
\end{tabular}

Four proteins downstream of Akt that are known to be involved in the intracellular actions of insulin signaling were investigated in the PNS of nondiabetic and $o b / o b$ mice. In both the DRG and sciatic nerve, there were no significant changes in the activation of mTor, p70S6K, or AS160 in either nondiabetic or ob/ob mice, nor was there a significant change in the inhibition of GSK3 $\beta$. Data presented is the fold change of protein modification (measured with Western blot analysis) induced by insulin as compared to that observed in mice that received PBS. $n=7-10$ for all groups. 


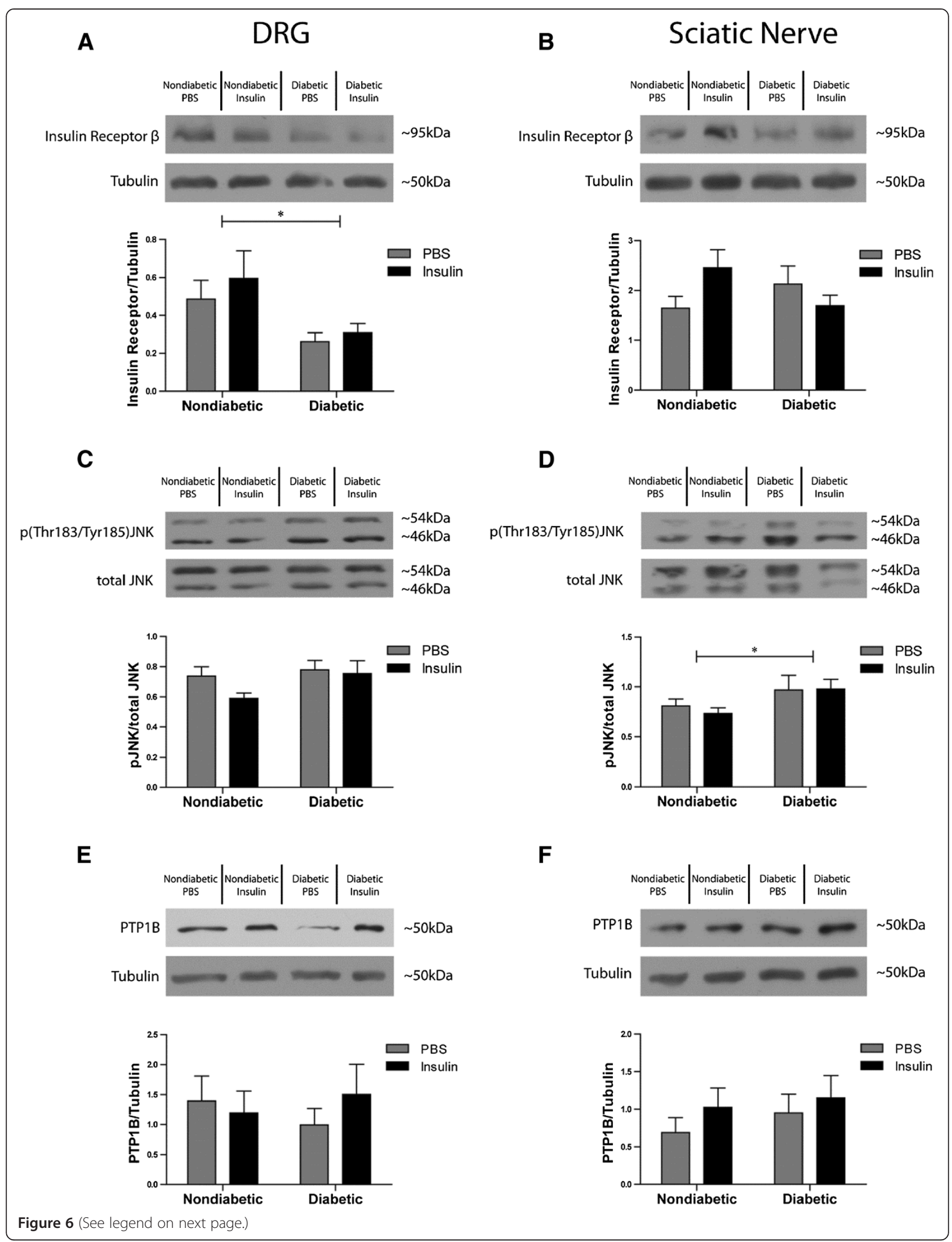


recognized mechanism of insulin resistance in muscle and adipose. In the current study, we investigated both IRS1 (muscle and adipose isoform) [41] and IRS2 (neural isoform) $[6,42]$ serine phosphorylation. In contrast to neurons in vitro, IRS serine phosphorylation does not appear to be significantly affected in the PNS in vivo within this model, (data not shown). Interestingly, there was significant activation of the stress kinase JNK (p(Thr183/Tyr185)JNK/total JNK) in the sciatic nerve of $o b / o b$ mice compared to nondiabetic mice (Figure 6D) and a similar pattern of activated JNK was observed in the DRG of $o b / o b$ mice, however significance was not reached (nondiabetic vs. diabetic $\mathrm{p}=0.122)$ (Figure 6C).

In addition to stress kinase activation and reduced insulin receptor expression, insulin resistance can also be induced by over activation of tyrosine phosphatases [29]. However, PTP1B expression was not elevated in the DRG or sciatic nerve of $o b / o b$ mice, nor did insulin stimulation appear to alter its expression levels (Figure 6E,F, respectively).

\section{Discussion}

Diabetic neuropathy is associated with profound loss of distal limb sensation and/or pain, causing significant decline in the quality of life and potential morbidity and mortality for patients. Currently, there are no clinical treatments that successfully improve neuropathic damage to peripheral sensory nerve fibers, likely due to the multifactorial etiology of neuropathy development and progression. Here, we have demonstrated in vivo PNS insulin resistance in $o b / o b$ mice. These results are consistent with recent in vitro studies and support the view that altered insulin signaling may contribute to DN [43]. A robust activation of insulin-sensitive pathways was observed in the DRG and sciatic nerve of nondiabetic mice, with a blunted response in both tissues from insulin-resistant $o b / o b$ mice. While no one mechanism of insulin resistance was clearly prevalent, significant changes were seen in two known pathways of insulin resistance, including increased JNK activity and reduced insulin receptor expression. Although more research is needed to fully elucidate the pathways leading to PNS insulin resistance, these results suggest that cellular mechanisms of insulin resistance that have been defined in muscle may also play an important role in the PNS.
These experiments used an in vivo approach to support the mounting in vitro evidence pointing to PNS insulin resistance in diabetes. Interestingly, Akt activation was very prominent in the DRG and sciatic nerve of nondiabetic mice, yet very few significant changes were seen in downstream signaling molecules. This may be due to a temporal effect, as downstream mediators of the Akt pathway may have not yet been activated during the 30-minute stimulation period used for this study. However, it is also plausible that the downstream Akt signaling proteins explored in this study do not play a prominent role in insulin pathways within the DRG. Instead of driving protein synthesis through mTor and p70S6K or regulation of GSK3 $\beta$ actions, insulin may be playing a more important role in lipid and glucose metabolism, gene regulation, or mitochondrial maintenance in peripheral neurons. Further studies are underway to explore these other downstream components of insulin signaling and to define the temporal components of this signaling pathway.

An additional caveat to this study is the use of leptindeficient $o b / o b$ mice. Leptin's role in the nervous system is receiving increasing attention, and it may have a neuroprotective role [44]. It is not known how reduced neuronal leptin may have contributed to our results. Thus, confirming these results in a high-fat diet model of obesity will be an important step to further investigating PNS insulin resistance.

In experiments presented here, it appeared that insulin produced a stronger Akt activation in the sciatic nerve compared to the DRG (Figure 3), whereas IGF1 produced a stronger Akt activation in the DRG compared to the sciatic nerve (Figure 5). These results point to an apparent separation in insulin/IGF-1 signaling support within the PNS. One plausible explanation may be that insulin and IGF-1 have different actions on the DRG soma and satellite cells compared to sensory axons, motor axons, and Schwann cells in the peripheral nerve, leading to alternative signaling profiles. How this potential divergence in signaling may affect sensory neuron function is yet to be determined and ongoing research is further delineating the differential roles that insulin and IGF-1 may play in sensory nerve biology.

In $o b / o b$ mice, both the DRG and sciatic nerve displayed reduced insulin-induced Akt activation, a classic indication 
of insulin resistance. Several mechanisms of insulin resistance outlined in muscle also appear to be altered in the PNS, and may be contributing to the observed reduction in insulin signal transduction. However, these results must be interpreted with caution as significant changes were not seen consistently across PNS tissues, and further research will need to be completed to fully establish a clear mechanism. Interestingly, no change in baseline Akt activation levels was observed between nondiabetic and $o b / o b$ mice as may be expected in states of insulin resistance. These results are intriguing and suggest that future research focusing on pathways driving Akt signaling is warranted.

Hyperinsulinemia can promote insulin resistance through downregulation of the insulin receptor [22]. This effect was demonstrated in our data. The $o b / o b$ mice in this cohort had serum insulin levels 34.3 fold higher than nondiabetic mice and the DRG of $o b / o b$ mice displayed significantly lower insulin receptor expression. Thus, the extreme hyperinsulinemia in the $o b / o b$ mice may be promoting insulin receptor downregulation and contributing to PNS insulin resistance. This idea is supported by a recent study that reported a significant decrease in insulin receptor mRNA in cultured DRG neurons that displayed insulin resistance when treated with high levels of insulin [7].

An alternative mediator of insulin resistance is the stress kinase JNK, which is activated in response to various cellular stressors, including low grade chronic inflammation induced by obesity $[33,45]$. In fact, $o b / o b$ mice with a JNK null mutation have improved whole body glucose tolerance and insulin sensitivity [31]. Additionally, JNK activation has been implicated in altered neurofilament phosphorylation in the PNS of diabetic rats [46]. JNK activation is proposed to promote insulin resistance through upregulation of IRS serine phosphorylation, and IRS is a key common signaling component of both the insulin and IGF-1 pathways. In the current study we observed increased JNK activation without a significant elevation in either IRS1 or IRS2 serine phosphorylation. Some controversy does exist as to which serine sites are most important in insulin resistance, thus the serine sites that we probed ( $p($ ser731)IRS2 and ( $\mathrm{p}($ ser307)IRS1) may not be heavily involved in inhibiting insulin signaling in the PNS. More powerful approaches, such as mass spectrometry, may be needed to establish a global change in the IRS phosphorylation profile within the PNS [47].

Another possible component of the insulin receptor signaling pathway that could be affected in insulin resistance is PTP1B. PTP1B is the canonical member of protein tyrosine phosphatases and serves an important role in insulin signaling regulation [29]. Overexpression of $\mathrm{PTP} 1 \mathrm{~B}$ has been linked to insulin resistance in peripheral tissues of $o b / o b$ mice [26] and
PTP1B knockout mice display increased insulin sensitivity [48]. In the current study, we did not detect significant upregulation of PTP1B in the DRG or sciatic nerve of insulin resistant mice. While there was no change in PTP1B expression, there still could be alterations in phosphatase activity and further studies are underway to explore this possibility.

It will be important to put the current results in context with other contributory mechanisms of DN, including glucose and/or lipid mediated toxicity as well as oxidative stress [49]. We postulate that the metabolic dysfunction associated with hyperglycemia and dyslipidemia in concert with reduced neurotrophic support promotes deterioration and reduced regeneration of the distal axon. Furthermore, the loss of appropriate insulin signaling could make neurons even more susceptible to these pathogenic cascades. It should be noted that both intrathecal and intraperitoneal insulin injections altered blood glucose levels. Thus, these results should be viewed in the context that glucose levels were also transiently altered by insulin administration. Further research into disrupted PNS insulin signaling relative to other pathogenic mechanisms is needed, as this will be a key step in translating these basic science results into clinical applications.

\section{Conclusions}

Insulin resistance is emerging as a potential mediator of several neurological syndromes (reviewed in [1]). This study, along with recent data of in vitro DRG insulin resistance, strongly supports altered insulin signaling as a pathogenic mechanism in DN. While deficient insulin signaling has been a proposed contributor to DN in type 1 models for some time $[3,4,14,43,50]$, little has been known about insulin signaling effectiveness in type 2 (hyperinsulinemic) models of DN. We observed reduced insulin signaling in vivo in the PNS of type 2 diabetic $o b / o b$ mice and suggest possible mechanisms that may be contributing to these changes. It is now becoming evident that decreased insulin neurotrophic support in the PNS is an integral part of DN and may be a congruent mechanism between type 1 and type 2 diabetic models of $\mathrm{DN}$, as both have reduced insulin signaling either due to insulinopenia or neuronal insulin resistance.

Future studies will focus on mechanisms through which insulin supports proper PNS function, as revealing these pathways may provide insight into how decreased insulin support contributes to the pathogenesis of DN. Furthermore, delineating the details of PNS insulin signaling may open new avenues for therapeutic intervention in patients with $\mathrm{DN}$. 


\section{Methods \\ Animals}

All experiments were approved by the University of Kansas Medical Center Institutional Animal Care and Use Committee. Male ob/ob leptin null mutant and agematched control mice $(\mathrm{ob} /+)$ were purchased from Jackson Laboratories (Bar Harbor, Maine) at 8 weeks of age. Mice were given access to food and water ad libitum and housed on a 12-hour light/dark cycle. Weekly blood glucose (Glucose Diagnostic Assay Sigma-Aldrich, St. Louis, $\mathrm{MO}$ ), serum insulin (Insulin ELISA Alpco, Salem, NH) and weights were monitored and mice were sacrificed at 11 weeks of age.

\section{Glucose tolerance test}

At 9 weeks of age, an intraperitoneal glucose tolerance test (IPGTT) was used to assess the response of mice to a glucose challenge. After a 6-hour fast, mice were given an intraperitoneal injection of glucose at $1 \mathrm{~g}$ of glucose per kg body weight. Blood glucose levels were measured via tail clip immediately prior to the glucose bolus and then at $15,30,60$, and 120 minutes after injection.

\section{Insulin tolerance test}

At 10 weeks of age, mice underwent an insulin tolerance test (ITT). Mice were fasted for 6 hours and then administered IP insulin (Humulin R, Lilly, Indianapolis, Indiana) at a dosage of $1.5 \mathrm{U}$ per $\mathrm{kg}$ body weight. Blood glucose levels were monitored immediately prior to insulin injection and then at 15, 30, 60, and 120 minutes thereafter.

\section{HOMA-IR}

Fasting insulin and fasting glucose levels were used to calculate the homeostatic model assessment of insulin resistance (HOMA-IR). Scores were calculated with the following equation: (blood glucose $(\mathrm{mg} / \mathrm{dl}) \mathrm{X}$ (serum insulin $(\mathrm{uU} / \mathrm{mL})) / 405)[51]$.

\section{Mechanical sensitivity}

Mechanical behavioral responses to Semmes Weinstein-von Frey monofilaments $(0.07$ to $5.0 \mathrm{~g})$ were assessed at 8 , 9,10 , and 11 weeks of age. Mice underwent acclimation 2 days prior to the first day of behavioral testing. Mice were placed in individual clear plastic cages $(11 \times 5 \times 3.5 \mathrm{~cm})$ on a wire mesh grid $55 \mathrm{~cm}$ above the table and were acclimated for 30 minutes prior to behavioral analysis. The filaments were applied perpendicularly to the plantar surface of the hindpaw until the filament bent. Testing began with the $0.7 \mathrm{~g}$ filament, and in the presence of a response, the next smaller filament was applied. If no response was observed, the next larger filament was used. Filaments were applied until there was a change in response, followed by an additional 4 more applications. The withdrawal threshold was calculated using the formula from the up-down method previously described [52].

\section{Insulin and IGF-1 injections}

Sterile PBS (vehicle), $0.1 \mathrm{U}(\sim 0.7 \mathrm{nmol})$ Humulin R insulin, or recombinant IGF-1 equimolar to $0.1 \mathrm{U}$ insulin was directly administered to both nondiabetic and $o b / o b$ type 2 diabetic mice via a one-time intrathecal injection. Previously, intrathecal $0.1 \mathrm{U}$ insulin and equimolar IGF-1 have been shown to have beneficial effects on the symptoms of DN [10]. All injections were $50 \mu \mathrm{L}$ and administered with a $1 \mathrm{cc} 28 \frac{1}{2}$ gauge insulin syringe between the L6 and S1 vertebrae. In an additional preliminary study, sterile PBS or insulin was delivered through an intraperitoneal injection at a dose of $3.33 \mathrm{U} / \mathrm{kg}$, such that the total insulin administered was approximately $0.1 \mathrm{U}$ for nondiabetic mice and $0.17 \mathrm{U}(\sim 1.2 \mathrm{nmol})$ for $o b / o b$ mice. The doses administered and stimulation time frames used were confirmed to be sufficient for Akt activation in the PNS with dose curve and time course studies (Grote, unpublished observation).

\section{Western blots}

After a 30 minute insulin stimulation period, the right and left lumbar DRG and sciatic nerves were harvested for each sample from 11 week old mice and frozen at $-80^{\circ} \mathrm{C}$. Tissues were sonicated in Cell Extraction Buffer (Invitrogen, Carlsbad, CA) containing $55.55 \mu \mathrm{l} / \mathrm{ml}$ protease inhibitor cocktail, $200 \mathrm{mM} \mathrm{Na}_{3} \mathrm{VO}_{4}$, and $200 \mathrm{mM} \mathrm{NaF}$. Following sonication, protein was extracted on ice for 60 minutes and vortexed every 10 minutes. After centrifugation, protein concentration of the supernatant was measured with a Bradford assay (Bio-Rad, Hercules, CA). Samples were then boiled with Lane Marker Reducing Sample Buffer (Thermo Scientific, Waltham, MA) for 3 minutes. Equal amounts of protein $(30 \mu \mathrm{g})$ were loaded per lane and samples were separated on a $4-15 \%$ gradient tris-glycine gel (Bio-Rad), and then transferred to a nitrocellulose membrane. Membranes were probed with the following primary antibodies and all antibodies were purchased from Cell Signaling (Danvers, MA) unless otherwise noted: total Akt (1:2000), p-(Ser473)Akt (1:500), total p70S6K (1:500), p-(Thr389)p70S6K (1:500), total GSK3 $\beta$ (1:1500), p-(Ser9)GSK3 $\beta$ (1:1000), total JNK (1:1000), p-(Thr183/Tyr185)JNK (1:500), total mTor (1:500), p-(Ser2448)mTor (1:500), Insulin-like growth factor 1 receptor $\beta$ subunit (1:500), PTP1B (1:500) (Abcam, Cambridge, MA), total AS160 (1:1000) (Millipore, Billerica, MA), p-(Thr642)AS160 (1:500) (Millipore), Insulin Receptor $\beta$ subunit (1:500) (Santa Cruz, Santa Cruz, CA), and $\alpha$-tubulin (1:5000) (Abcam). Bands were visualized with either anti-mouse or anti-rabbit HRP-conjugated secondary antibodies (Santa Cruz) and ECL with X-ray film. 
Densitometry with ImageJ (NIH) was then used to analyze each lane. All samples from each tissue were run simultaneously across multiple gels and each group was equally represented on each gel (approximately 3 nondiabetic PBS, 3 nondiabetic insulin, $3 o b / o b$ PBS, and $3 o b / o b$ insulin per gel). Data is presented as the ratio of integrated density of the phosopho-protein normalized to the integrated density of the total protein. The normalized ratio was averaged for each group and the mean \pm SEM is represented in the corresponding figures. Representative immunoblots are shown.

\section{Statistical analysis}

All data is expressed as means \pm standard error of the mean. IPGTT, ITT, and behavior data were analyzed with a repeated measures analysis of variance (RM-ANOVA). In addition, the area under the curve (AUC) for IPGTT and ITT was analyzed using a Student's $t$-test. Blood glucose changes at 30 minutes in response to insulin or IGF-1 were analyzed with a paired Student's $t$-test. Western blot results were analyzed with 2-way ANOVA and Bonferroni's post hoc analysis when appropriate. Outliers greater than or less than 2 standard deviations from the mean were not included in the analysis. All statistical tests were performed using SigmaPlot software and a $P$ value $<0.05$ was considered significant.

\section{Abbreviations \\ CNS: Central nervous system; PNS: Peripheral nervous system; IRS: Insulin receptor substrate; mTor: Mammalian target of rapamycin; GSK3ß: Glycogen synthase kinase 3 beta; MAPK: Mitogen-activated protein kinase; PTP1B: Protein tyrosine phosphatase 1B; JNK: c-Jun N-terminal kinase; DN: Diabetic neuropathy.}

\section{Competing interests}

The authors have no competing interests to report.

\section{Authors' contributions}

CG carried out the Western blot analysis, participated in metabolic characterization of mice, performed the statistical analysis and drafted the manuscript. AG carried out the behavior analysis of mechanical sensitivity. JR performed intrathecal injections, tissue dissections, and participated in metabolic characterization of mice. PG helped conceive the study and participated in the design of the study. EF participated in data interpretation and experimental design and helped draft the manuscript. DW conceived the study, and participated in its design and coordination and helped to draft the manuscript. All authors read and approved the final manuscript.

\section{Acknowledgements}

This work was supported by National Institutes of Health $(\mathrm{NIH})$ grants R01NS43313 (DEW), R01AG031575 (PCG), and P20GM103418 from the Kansas Idea Network of Biomedical Research Excellence (K-INBRE) program of the National Institute of General Medical Sciences, and by the Kansas IDDRC, P30 NICHD HD 002528.

\section{Author details}

'Department of Anatomy and Cell Biology, the University of Kansas Medical Center, Kansas City, KS 66160, USA. ${ }^{2}$ Department of Molecular and Integrative Physiology, the University of Kansas Medical Center, Kansas City, KS 66160, USA. ${ }^{3}$ Department of Neurology, University of Michigan, Ann Arbor, MI 48109, USA.
Received: 20 March 2013 Accepted: 19 April 2013

Published: 10 May 2013

\section{References}

1. Kim B, Feldman EL: Insulin resistance in the nervous system. Trends Endocrinol Metab 2012, 23(3):133-141.

2. Singhal $A$, et al: Near nerve local insulin prevents conduction slowing in experimental diabetes. Brain Res 1997, 763(2):209-214.

3. Brussee V, Cunningham FA, Zochodne DW: Direct insulin signaling of neurons reverses diabetic neuropathy. Diabetes 2004, 53(7):1824-1830.

4. Toth C, Brussee V, Zochodne DW: Remote neurotrophic support of epidermal nerve fibres in experimental diabetes. Diabetologia 2006, 49(5):1081-1088.

5. Kim B, et al: Hyperinsulinemia Induces Insulin Resistance in Dorsal Root Ganglion Neurons. Endocrinology 2011, 152(10):3638-3647.

6. Grote $\mathrm{CW}$, et al: Insulin receptor substrate 2 expression and involvement in neuronal insulin resistance in diabetic neuropathy. Exp Diabetes Res 2011, 2011:212571.

7. Singh $B$, et al: Resistance to trophic neurite outgrowth of sensory neurons exposed to insulin. J Neurochem 2012, 121(2):263-276.

8. Lee $\mathrm{KO}$, et al: Insulin resistance is independently associated with peripheral and autonomic neuropathy in Korean type 2 diabetic patients. Acta Diabetol 2012, 49(2):97-103.

9. Patel NJ, et al: Glucose and leucine uptake by rat dorsal root ganglia is not insulin sensitive. J Neurol Sci 1994, 121(2):159-162.

10. Toth $C$, et al: Rescue and regeneration of injured peripheral nerve axons by intrathecal insulin. Neuroscience 2006, 139(2):429-449.

11. Guo $G$, et al: Local insulin and the rapid regrowth of diabetic epidermal axons. Neurobiol Dis 2011, 43(2):414-421.

12. Recio-Pinto $E$, Rechler MM, Ishii DN: Effects of insulin, insulin-like growth factor-II, and nerve growth factor on neurite formation and survival in cultured sympathetic and sensory neurons. I Neurosci 1986, 6(5):1211-1219.

13. Fernyhough $P$, et al: Insulin and insulin-like growth factor $I$ enhance regeneration in cultured adult rat sensory neurones. Brain Res 1993, 607(1-2):117-124.

14. Huang $T J$, et al: Insulin prevents depolarization of the mitochondrial inner membrane in sensory neurons of type 1 diabetic rats in the presence of sustained hyperglycemia. Diabetes 2003, 52(8):2129-2136.

15. Chowdhury SK, et al: Mitochondrial respiratory chain dysfunction in dorsal root ganglia of streptozotocin-induced diabetic rats and its correction by insulin treatment. Diabetes 2010, 59(4):1082-1091.

16. McNay EC, et al: Hippocampal memory processes are modulated by insulin and high-fat-induced insulin resistance. Neurobiol Learn Mem 2010, 93(4):546-553.

17. Craft S, et al: Intranasal Insulin Therapy for Alzheimer Disease and Amnestic Mild Cognitive Impairment: A Pilot Clinical Trial. Arch Neurol 2012, 69(1):29-38.

18. Gerozissis K: Brain insulin, energy and glucose homeostasis; genes, environment and metabolic pathologies. Eur J Pharmacol 2008, 585(1):38-49.

19. Obici $S$, et al: Hypothalamic insulin signaling is required for inhibition of glucose production. Nat Med 2002, 8(12):1376-1382.

20. Le Roith $D$, Zick Y: Recent advances in our understanding of insulin action and insulin resistance. Diabetes Care 2001, 24(3):588-597.

21. Cheng Z, Tseng Y, White MF: Insulin signaling meets mitochondria in metabolism. Trends Endocrinol Metab 2010, 21(10):589-598.

22. Mayer CM, Belsham DD: Central insulin signaling is attenuated by longterm insulin exposure via insulin receptor substrate-1 serine phosphorylation, proteasomal degradation, and lysosomal insulin receptor degradation. Endocrinology 2010, 151(1):75-84.

23. Gavin JR 3rd, et al: Insulin-dependent regulation of insulin receptor concentrations: a direct demonstration in cell culture. Proc Natl Acad Sci U S A 1974, 71(1):84-88.

24. Shanik MH, et al: Insulin resistance and hyperinsulinemia: is hyperinsulinemia the cart or the horse? Diabetes Care 2008, 31(Suppl 2):S262-S268.

25. Garvey WT, Olefsky JM, Marshall S: Insulin receptor down-regulation is linked to an insulin-induced postreceptor defect in the glucose transport system in rat adipocytes. $J$ Clin Invest 1985, 76(1):22-30. 
26. Gum RJ, et al: Reduction of protein tyrosine phosphatase $1 \mathrm{~B}$ increases insulin-dependent signaling in ob/ob mice. Diabetes 2003, 52(1):21-28.

27. Koren S, Fantus IG: Inhibition of the protein tyrosine phosphatase PTP1B: potential therapy for obesity, insulin resistance and type- 2 diabetes mellitus. Best Pract Res Clin Endocrinol Metab 2007, 21(4):621-640.

28. Nieto-Vazquez I, et al: Protein-tyrosine phosphatase 1B-deficient myocytes show increased insulin sensitivity and protection against tumor necrosis factor-alpha-induced insulin resistance. Diabetes 2007 56(2):404-413.

29. Kenner KA, et al: Protein-tyrosine phosphatase $1 \mathrm{~B}$ is a negative regulator of insulin- and insulin-like growth factor-l-stimulated signaling. J Biol Chem 1996, 271(33):19810-19816.

30. Paz K, et al: A molecular basis for insulin resistance. Elevated serine/ threonine phosphorylation of IRS- 1 and IRS- 2 inhibits their binding to the juxtamembrane region of the insulin receptor and impairs their ability to undergo insulin-induced tyrosine phosphorylation. J Biol Chem 1997, 272(47):29911-29918.

31. Hirosumi J, et al: A central role for JNK in obesity and insulin resistance. Nature 2002, 420(6913):333-336.

32. Tanti JF, Jager J: Cellular mechanisms of insulin resistance: role of stressregulated serine kinases and insulin receptor substrates (IRS) serine phosphorylation. Curr Opin Pharmacol 2009, 9(6):753-762.

33. Lee $\mathrm{YH}$, et al: c-Jun $\mathrm{N}$-terminal kinase (JNK) mediates feedback inhibition of the insulin signaling cascade. J Biol Chem 2003, 278(5):2896-2902.

34. Aguirre $V$, et al: The c-Jun $\mathrm{NH}(2)$-terminal kinase promotes insulin resistance during association with insulin receptor substrate- 1 and phosphorylation of Ser(307). J Biol Chem 2000, 275(12):9047-9054.

35. Boura-Halfon S, Zick Y: Phosphorylation of IRS proteins, insulin action, and insulin resistance. Am J Physiol Endocrinol Metab 2009, 296(4):E581-E591.

36. Drel VR, et al: The leptin-deficient (ob/ob) mouse: a new animal model of peripheral neuropathy of type 2 diabetes and obesity. Diabetes 2006, 55(12):3335-3343.

37. Kim B, et al: Cortical neurons develop insulin resistance and blunted Akt signaling: a potential mechanism contributing to enhanced ischemic injury in diabetes. Antioxid Redox Signal 2011, 14(10):1829-1839.

38. Gupte AA, Bomhoff GL, Geiger PC: Age-related differences in skeletal muscle insulin signaling: the role of stress kinases and heat shock proteins. J Appl Physiol 2008, 105(3):839-848.

39. Bitar MS, Al-Mulla F: ROS constitute a convergence nexus in the development of IGF1 resistance and impaired wound healing in a rat model of type 2 diabetes. Dis Model Mech 2012, 5(3):375-388.

40. Talbot $K$, et al: Demonstrated brain insulin resistance in Alzheimer's disease patients is associated with IGF-1 resistance, IRS-1 dysregulation, and cognitive decline. J Clin Invest 2012, 122(4):1316-1338

41. Kahn CR: Knockout mice challenge our concepts of glucose homeostasis and the pathogenesis of diabetes. Exp Diabesity Res 2003, 4(3):169-182.

42. Schubert $M$, et al: Insulin receptor substrate-2 deficiency impairs brain growth and promotes tau phosphorylation. J Neurosci 2003, 23(18):7084-7092.

43. Ishii DN: Implication of insulin-like growth factors in the pathogenesis of diabetic neuropathy. Brain Res Brain Res Rev 1995, 20(1):47-67.

44. Dicou E, Attoub S, Gressens P: Neuroprotective effects of leptin in vivo and in vitro. Neuroreport 2001, 12(18):3947-3951.

45. Nguyen MT, et al: JNK and tumor necrosis factor-alpha mediate free fatty acid-induced insulin resistance in 3T3-L1 adipocytes. J Biol Chem 2005 280(42):35361-35371.

46. Fernyhough $P$, et al: Aberrant neurofilament phosphorylation in sensory neurons of rats with diabetic neuropathy. Diabetes 1999 48(4):881-889.

47. Langlais $P$, et al: Global IRS-1 phosphorylation analysis in insulin resistance. Diabetologia 2011, 54(11):2878-2889.

48. Elchebly $M$, et al: Increased insulin sensitivity and obesity resistance in mice lacking the protein tyrosine phosphatase-1B gene. Science 1999 283(5407):1544-1548.

49. Vincent $A M$, et al: Diabetic neuropathy: cellular mechanisms as therapeutic targets. Nat Rev Neurol 2011, 7(10):573-583.
50. Murakawa $Y$, et al: Impaired glucose tolerance and insulinopenia in the GK-rat causes peripheral neuropathy. Diabetes Metab Res Rev 2002, 18(6):473-483.

51. Kim MK, et al: PAR-5359, a well-balanced PPARalpha/gamma dual agonist, exhibits equivalent antidiabetic and hypolipidemic activities in vitro and in vivo. Eur J Pharmacol 2008, 595(1-3):119-125.

52. Chaplan SR, et al: Quantitative assessment of tactile allodynia in the rat paw. J Neurosci Methods 1994, 53(1):55-63.

doi:10.1186/2051-5960-1-15

Cite this article as: Grote et al:: Peripheral nervous system insulin resistance in ob/ob mice. Acta Neuropathologica Communications 2013 $1: 15$.

\section{Submit your next manuscript to BioMed Central and take full advantage of:}

- Convenient online submission

- Thorough peer review

- No space constraints or color figure charges

- Immediate publication on acceptance

- Inclusion in PubMed, CAS, Scopus and Google Scholar

- Research which is freely available for redistribution 